\title{
PATHOLOGICAL TOPOLOGIES AND RANDOM WALKS ON ABELIAN GROUPS
}

\author{
R. M. DUDLEY ${ }^{1}$
}

It is shown in this paper that a part of the structure theory of locally compact abelian groups, which does not explicitly involve compactness, still fails to apply to complete separable metric abelian groups. Specifically, Theorem 2 asserts that any countable abelian group may be discretely imbedded in a metrizable abelian group with a dense cyclic subgroup. This result is independent of the rest of the paper.

Theorem 1 is a natural generalization of the result of [4], on the existence of recurrent random walks on discrete abelian groups, to locally compact abelian groups. Theorem 2 shows that it cannot be applied verbatim to complete separable metric groups. However, a statement in terms of dense subgroups rather than discrete ones is formulated as a conjecture.

We call a nonnegative finite measure $\mu$ defined on the Borel sets (the $\sigma$-field generated by the open sets) of a topological space regular if for every Borel set $B$ and $\epsilon>0$ there is a compact set $K \subset B$ such that $\mu(B)-\mu(K)<\epsilon$. It is known that any finite Borel measure on a complete separable metric space is regular, and that assuming the generalized continuum hypothesis "separable" is unnecessary (see [6]). Also, a bounded linear functional on the continuous functions on a compact Hausdorff space may be uniquely represented as the integral with respect to a regular Borel measure (see $[5, \S 56]$ ).

In order to define random walks on topological groups, we must define convolution of measures. Let $G$ be a Hausdorff topological group and let $\mu$ and $\nu$ be finite regular Borel measures on $G$. Then there are disjoint compact subsets $C_{1}, C_{2}, \cdots$, of $G$ such that both $\mu$ and $\nu$ are concentrated in $\bigcup_{n=1}^{\infty} C_{n}$.

To obtain a product measure for $\mu$ and $\nu$ on $G \times G$, defined on all Borel sets, we may proceed as follows. On each compact set $C_{m} \times C_{n}$ we have a positive linear functional

$$
f \rightarrow \int_{C_{n}} \int_{C_{m}} f(x, y) d \mu(x) d \nu(y)
$$

Received by the editors August 21, 1962 and, in revised form, January 11, 1963.

${ }^{1}$ Some of the work on this paper was done while the author held a National Science Foundation Cooperative Fellowship in 1961-1962. 
on the continuous functions on $C_{m} \times C_{n}$. (It follows from the StoneWeierstrass theorem that the order of integration can be reversed.) This linear functional can, as mentioned, be written as

$$
f \rightarrow \int f d \rho_{m n}
$$

where $\rho_{n m}$ is a regular Borel measure of total mass $\mu\left(C_{m}\right) \nu\left(C_{n}\right)$ on $C_{m} \times C_{n}$. The measure $\sum_{m, n=1}^{\infty} \rho_{m n}$ is then a regular Borel measure on $G \times G$ which is easily seen by mutual refinement to be independent of the choice of the sets $C_{n}$. We call this measure $\mu \otimes \nu$. It is easily seen to be an extension of the ordinary product measure $\mu \times \nu$.

If there is no countable base for the topology of $G$, it seems unclear whether $\mu \times \nu$ itself is defined on all Borel sets in $G \times G$, even after completion of the measure, a point not explained in [8]. Another way of obtaining a sufficiently widely defined product measure $\mu \nu$ is given in [1]. Since the $\mu \nu$ measure of any Borel set $B$ is the supremum of the measures of closed sets included in $B,{ }^{2}$ and for any closed set $F$

$$
\mu \nu(F)=\sum_{m, n=1}^{\infty} \mu \nu\left(F \cap\left(C_{m} \times C_{n}\right)\right),
$$

$\mu \nu$ is regular. $\mu \nu$ has the property that

$$
\int f d \mu \nu=\iint f(x, y) d \mu(x) d \nu(y)
$$

for any Borel function $f$ on $G \times G$. Applying this to functions equal to continuous functions on $C_{m} \times C_{n}$ and zero elsewhere, it follows that $\mu \nu$ and $\mu \otimes \nu$ agree on all Borel sets.

If $\psi$ is a continuous mapping of a topological space $A$ onto another space $B$ and $\mu$ is a regular Borel measure on $A$, we can define a regular Borel measure $\mu_{\psi}$ on $B$ by $\mu_{\psi}(M)=\mu\left(\psi^{-1}(M)\right)$ for any Borel set $M$ in $B$ (see [8]). If $g$ is a bounded Borel function on $B$ and $M$ is a Borel set in $B$, then

$$
\int_{M} g d \mu_{\psi}=\int_{\psi^{-1}(M)} g(\psi(x)) d \mu(x)
$$

since the equality holds for functions with finitely many values.

Now, if $p(x, y)=x y$ on $G \times G$, we define the convolution $\mu * \nu$ by

$$
\mu * \nu=(\mu \otimes \nu)_{p}=(\mu \nu)_{p}
$$

2 This property is stated in [1] only for $B$ open, but since it also holds for $B$ closed and for countable unions and intersections of sets where it holds, it is true of all Borel sets. 
Then $\mu * \nu$ is a regular Borel measure on $G$ satisfying, and characterized by,

$$
\begin{aligned}
\int f d(\mu * \nu) & =\int f(x y) d \mu \nu(x, y)=\iint f(x y) d \mu(x) d \nu(y) \\
& =\iint f(x y) d \nu(y) d \mu(x)
\end{aligned}
$$

for any bounded Borel function $f$. It follows directly that convolution is associative and is commutative if $G$ is abelian. We also have

$$
(\mu * \nu)(B)=\int \mu\left(B y^{-1}\right) d \nu(y)=\int \nu\left(x^{-1} B\right) d \mu(x)
$$

for any Borel set $B$, as in [8]. If the topology of $G$ has a countable base, all these facts can be proved without recourse to [1] since we need only apply Fubini's theorem for the ordinary product measure.

Here is a fact relating convolution and the map $\mu \rightarrow \mu_{\psi}$ :

Lemma 1. If $G$ and $H$ are topological groups, $\psi$ is a continuous homomorphism of $G$ into $H$, and $\mu$ and $\nu$ are regular Borel measures on $G$, then

$$
(\mu * \nu)_{\psi}=\mu_{\psi} * \nu_{\psi} \text {. }
$$

Proof. If $B$ is a Borel set in $H$,

$$
\begin{aligned}
\left(\mu_{\psi} * \nu_{\psi}\right)(B) & =\int_{H} \mu_{\psi}\left(B h^{-1}\right) d \nu_{\psi}(h) \\
& =\int_{G} \mu_{\psi}\left(B \psi(y)^{-1}\right) d \nu(y) \\
& =\int_{G} \mu\left(\psi^{-1}\left(B \psi\left(y^{-1}\right)\right)\right) d \nu(y) \\
& =\int_{G} \mu\left(\psi^{-1}(B) y^{-1}\right) d \nu(y) \\
& =(\mu * \nu)\left(\psi^{-1}(B)\right)=(\mu * \nu)_{\psi}(B), \quad \text { q.e.d. }
\end{aligned}
$$

To define a "random walk" on an abelian topological group $G$, we will have regular Borel probability measures $\mu$ and $\nu$ on $G$, where $\nu$ is an "initial distribution" and $\mu$ defines a "transition probability." Thus we want to define a probability measure $\operatorname{Pr}$ on the infinite product

$$
G^{\omega}=G \times G \times G \cdots=G_{0} \times G_{1} \times G_{2} \cdots
$$


or space of sequences $\left\{x_{n}: n=0,1,2, \cdots, x_{n} \in G\right\}$ such that the variables $x_{0}, x_{1}-x_{0}, \cdots, x_{n+1}-x_{n}, \cdots$ are all independent and $\operatorname{Pr}\left(x_{0} \in B\right)=\nu(B), \operatorname{Pr}\left(x_{n+1}-x_{n} \in B\right)=\mu(B)$ for any $n \geqq 0$ and Borel set $B \subset G$.

To do this, we first define a product measure $\nu \otimes \mu \otimes \mu \otimes \cdots$ on $G^{\omega}$. If $H_{n}=G_{0} \times G_{1} \times \cdots \times G_{n}$, then we have a regular Borel measure

$$
\nu \otimes \mu^{(n)}=\nu \otimes \mu \otimes \cdots \otimes \mu
$$

on $H_{n}$ for each $n$ (it is easy to check that the operation $\otimes$ is associative). For each $n$ there is a natural mapping $\psi_{n}$ of $H_{n+1}$ onto $H_{n}$, and clearly

$$
\left(\nu \otimes \mu^{(n+1)}\right)_{\psi_{n}}=\nu \otimes \mu^{(n)} .
$$

Thus all the hypotheses of a theorem of Bochner [2, Theorem 5.1.1, p. 120] on inverse limits of measures are satisfied. Hence there is a probability measure $P$ on $G^{\omega}$ such that if $\phi_{n}$ is the natural mapping of $G$ onto $H_{n}$ for any $n, P_{\phi_{n}}=\nu \otimes \mu^{(n)}$. It is clear that for any closed set $F \subset G^{\omega}$ (for the product topology),

$$
F=\bigcap_{n=1}^{\infty} \phi_{n}^{-1}\left(\phi_{n}(F)\right) .
$$

Thus $P$ is defined on all Borel sets in $G^{\omega}$. Since there are compact sets $\bigcap_{n-1}^{\infty} \phi_{n}^{-1}\left(K_{n}\right)$ in $G^{\omega}$, where $K_{n}$ is compact in $H_{n}$, with measure arbitrarily close to $1, P$ is regular on closed sets. It follows easily that $P$ is also regular on open sets and hence on all Borel sets.

Finally, let $R W$ be the mapping of $G^{\omega}$ into itself defined by

$$
R W\left(y_{0}, y_{1}, y_{2}, \cdots\right)=\left(y_{0}, y_{0}+y_{1}, y_{0}+y_{1}+y_{2}, \cdots\right) .
$$

$R W$ is clearly continuous, so that $\operatorname{Pr}=P_{R W}$ is a regular Borel measure on $G^{\omega}$. The measure space $\left(G^{\omega}, \operatorname{Pr}\right)$ will be called the random walk on $G$ with initial distribution $\nu$ and transition probability $\mu$. (If $G$ has a countable base for its topology, $P$ is the usual infinite product measure and our other definitions also coincide essentially with the usual ones.) A random walk will be called recurrent if for every open set $U \subset G$,

$$
\operatorname{Pr}\left(x_{n} \in U \text { for infinitely many } n\right)=1 .
$$

THeORem 1. A locally compact abelian group $G$ has a recurrent random walk if and only if (a) for every open subgroup $H$ of $G, G / H$ is countable, and (b) $G$ does not have a discrete subgroup free abelian on three generators. 
To prove Theorem 1 we shall first generalize Lemma 1 of [2]. If $\left(G^{\omega}, \operatorname{Pr}\right)$ is a recurrent random walk on $G$ and $U$ is an open subgroup of $G$, let $t_{0}$ be the least $n$ such that $x_{n} \in U, t_{1}$ the next least, and so on; each $t_{j}, j=0,1,2, \cdots$, is defined with probability one and equal to a Borel function on its domain. Let $x_{n}=x(n)$ and $y_{n}=x\left(t_{n}\right)$ for any $n$.

Now $y_{0}$ has a distribution $\nu_{U}$ and $y_{1}-y_{0}$ has a distribution $\mu_{U}$, where $\nu_{U}$ and $\mu_{U}$ are regular Borel measures on $G$. For given values of $t_{0}, \cdots, t_{n}, t_{n+1}=t_{n}+k$ if and only if $k$ is the least positive integer such that $x\left(t_{n}+k\right)-x\left(t_{n}\right) \in U$, since $U$ is a subgroup. The set of variables $x\left(t_{n}+k\right)-x\left(t_{n}\right), k>0$, is independent of $x\left(t_{0}\right), \cdots, x\left(t_{n}\right)$, by a standard argument. Thus $y_{n+1}-y_{n}$ is independent of $y_{0}, \cdots, y_{n}$ and has distribution $\mu_{U}$ for all $n$. Thus if $\mu_{n}$ is the distribution of $\left(y_{0}, \cdots, y_{n}\right)$ on $H_{n}$, the measures $\mu_{n}$ have an inverse limit $\operatorname{Pr}_{U}$ on $G^{\omega}$, concentrated in $U^{\omega}$, so that $\left(U^{\omega}, \operatorname{Pr}_{U}\right)$ is a well-defined random walk on $U$ with initial distribution $\nu_{U}$ and transition probability $\mu_{U}$. The map $\left\{x_{n}\right\} \rightarrow\left\{x\left(t_{n}\right)\right\}$ of $G^{\omega}$ into $U^{\omega}$ obviously carries $\operatorname{Pr}$ into $\operatorname{Pr}_{U}$.

Lemma 2. $\left(U^{\omega}, \operatorname{Pr}_{U}\right)$ is recurrent.

Proof. For any open set $V \subset U, V$ is open in $G$, so that

$$
\operatorname{Pr}\left\{x_{n} \in V \text { infinitely often }\right\}=1,
$$

and since if $x_{n} \in V, n=t_{m}$ for some $m$,

$$
\begin{aligned}
\operatorname{Pr}\left\{x\left(t_{m}\right) \in V \text { infinitely often }\right\} & \\
& =\operatorname{Pr}_{U}\left\{y_{m} \in V \text { infinitely often }\right\}=1, \quad \text { q.e.d. }
\end{aligned}
$$

Let us now prove Theorem 1, beginning with "only if." Suppose $G$ has a recurrent random walk. $G$ cannot have an open subgroup $H$ with $G / H$ uncountable since for each $n$ at most countably many cosets of $H$ can be reached with positive probability by time $n$. To prove (b), we use the fact that $G$ has an open subgroup $H$ of the form $C \oplus V$ where $C$ is compact and $V$ is a vector group (see [7, pp. 160$162]$; the restriction to second-countable topologies is removed in the second edition). Suppose $J$ were a discrete subgroup of $G$, free on three generators. The sum of the ranks of $G / H$ and $J \cap H$ is then at least 3, and the rank of $J \cap H$ is less than or equal to the dimension of $V$ as a real vector space, since $J$ is discrete. Let $F$ be a free abelian subgroup of $G / H$ of maximal rank, and let $U$ be the union of all cosets of $H$ belonging to $F$. Then $U$ is an open subgroup of $G$ of the form $C \oplus R^{k} \oplus Z^{m}$, where $R$ and $Z$ are the additive groups of the real numbers and the integers respectively and $k+m \geqq 3$. (The relative 
topology of $U$ is the product topology indicated by the direct sum since $G / H$ is discrete.) By Lemma 2, there exists a recurrent random walk on $U$. Applying the natural homomorphism $\psi$ of $U$ onto $R^{k} \oplus Z^{m}$ and using Lemma 1 , we obtain a recurrent random walk on $R^{k} \oplus Z^{m}$, $k+m \geqq 3$, which is impossible (see [3, p. 31]).

To prove "if," again let $H=C \oplus V$ be an open subgroup of $G$ with $C$ compact, $V$ a vector group, and $G / H$ countable. Since $G / H$ is discrete, (b) implies that the sum of the rank of $G / H$ and the dimension of $V$ is at most 2 .

Thus $G / C$ has a countable dense subgroup $M$ of rank at most 2 . According to [4], there is a random walk on $M$ recurrent on $M$ as a discrete group and hence also with its relative topology from $G / C$. Thus there is a recurrent random walk on $G / C$ with its transition probability $\mu$ concentrated in countably many points. We may assume $\mu$ has no mass at 0 , according to the proof in [4].

Let $\rho$ be a normalized Haar measure on $C$ and each of its cosets. Let $\xi$ be the "product" of $\mu$ and $\rho$ defined by

$$
\xi(B)=\int_{G / C} \rho(B \cap x) d \mu(x)
$$

for each Borel set $B$. (Since $\mu$ is purely atomic, any function is measurable for it.) Probably $\xi$ is the transition probability of a recurrent random walk, but the proof seems to be easier if we modify it slightly, letting $\sigma=\left(\xi+\rho_{0}\right) / 2$, where $\rho_{0}$ is $\rho$ confined to $C$.

It is easy to see that if a transition measure $\alpha$ defines a recurrent random walk on a group $K$ for some initial distribution, and $\delta$ is the unit mass at the identity of $K$, then $\lambda \alpha+(1-\lambda) \delta$ is also the transition probability for a recurrent random walk with the same initial distribution if $0<\lambda \leqq 1$. Thus $(\mu+\delta) / 2$ defines a recurrent random walk on $G / C$ for any initial distribution, recurrent on $M$ as a discrete group.

Let $U$ be any open subset of $G$, and let $A$ be a coset of $C$ such that $\rho(A \cap U)>0$ and $A \in M$. Then a random walk on $G$ with initial distribution concentrated at 0 and transition probability $\sigma$ has probability 1 of being in $A$ infinitely often since it induces a random walk on $G / C$ with transition probability $(\mu+\delta) / 2$. If $x_{n} \in A$, then $\operatorname{Pr}\left(x_{n+1} \in A \cap U\right)=\frac{1}{2} \rho(A \cap U)>0$ since $\mu$ has no mass at 0 . The events that $x_{n(m)+1} \in A \cap U$, where $n(m)$ is the $m$ th integer $n$ such that $x_{n} \in A$, are independent for different $m$. Thus

$\operatorname{Pr}\left(x_{n} \in U\right.$ infinitely often $)$

$$
\geqq \operatorname{Pr}\left(x_{n} \in A \cap U \text { infinitely often }\right)=1, \quad \text { q.e.d. }
$$


The following result shows that "locally compact" cannot be replaced by "complete separable metric" in Theorem 1 .

TheORem 2. Let $G$ be any countable abelian group. Then there is a Hausdorff topology $T$ on the group $A=Z \oplus G$ with a countable base for which it is a topological group, $Z$ is dense, and $G$ has discrete relative topology.

Proof. Let $\left\{g_{n}\right\}_{n=1}^{\infty}$ be an enumeration of $G$ in which every element occurs infinitely many times and let $u_{n}$ be the element $\left(2^{2^{n}}, g_{n}\right)$ of $A, n=1,2, \cdots$. For each $n$, let $U_{n}$ be the set of all elements of $A$ which can be written as finite sums $\sum a_{i} u_{i}$ where the $a_{i}$ are integers such that $\left|a_{i}\right| \leqq 2^{i-n}$ for all $i$. (Thus $a_{i}=0$ for $i<n$.)

Then we have $U_{n}=-U_{n}$ for all $n$ and $U_{n+1}+U_{n+1} \subset U_{n}$. Thus the $U_{n}$ form a neighborhood-base at 0 for a topology $J$ on $A$ making it a topological group (see [7, p. 55]). If $u \in U_{n}$ for some $n$, then $u=\sum a_{i} u_{i}$ with $a_{i}=0$ for $i \geqq k$ for some $k$. Thus $U_{k}+u \subset U_{n}$, so that the $U_{n}$ are actually open sets (belong to $I$ ).

The sets $U_{n}+a, a \in A, n=1,2, \cdots$, form a base for $J$. Hence to show that $Z$ is dense in $A$ for $J$ it suffices to show that for any $n$ and $a=(z, g)$ there is an integer $w$ such that $(w, 0) \in(z, g)+U_{n}$, or $(z-w, g) \in-U_{n}=U_{n}$. This is clear since $g=g_{r}$ for some $r \geqq n$ and $u_{r}=\left(2^{2^{r}}, g_{r}\right) \in U_{n}$; let $w=z-2^{2^{r}}$.

If $\sum_{j=1}^{N} a_{j} 2^{2^{j}}=0$ with $\left|a_{j}\right| \leqq 2^{j-1}$ for all $j, a_{j}$ integers, then $a_{j}=0$ for all $j$ since

while

$$
\left|\sum_{j=1}^{n} a_{j} 2^{2^{j}}\right| \leqq n \cdot 2^{n-1} \cdot 2^{2^{n}}<2^{2 n+2^{n}} \leqq 2^{2^{n+1}}
$$

$$
\left|\sum_{j=n+1}^{N} a_{j} 2^{2^{j}}\right| \geqq 2^{2^{n+1}} \text { if } a_{n+1} \neq 0 .
$$

Thus the only element of $U_{1}$ of the form $(0, g)$ is $(0,0)$ so that the subgroup $G$ of elements of this form has discrete relative topology. Finally if $u=(z, g) \neq(0,0)$, then $U_{n}$ and $U_{n}+u$ are disjoint if $0<|z|$ $<2^{2^{n}}$ or if $z=0, g \neq 0$, so that $J$ is Hausdorff. This completes the proof.

COROLlaRy. There is a topology for the free abelian group $Z^{4}$ on four generators for which it has a recurrent random walk, and in which there is a subgroup free on three generators with discrete relative topology.

Proof. Let $G=Z^{3}$ in Theorem 2 and recall that $Z$ has a recurrent random walk. 
To see that Theorem 1 fails for complete separable metric groups it suffices to take a completion of the group described in the corollary with its topology as given by Theorem 2. The uniformity of this topology is metrizable, and the completion has a natural structure of complete metric group with a dense cyclic subgroup and a discrete subgroup free on three generators.

One might conjecture, however, that a complete abelian topological group has a recurrent random walk if and only if it has a compact subgroup whose quotient has a dense subgroup of rank at most 2 . "If" follows from the proof of Theorem 1.

I would like to thank the referee for suggesting a number of improvements and corrections in the proofs of both theorems.

\section{REFERENCES}

1. W. W. Bledsoe and A. P. Morse, Product measures, Trans. Amer. Math. Soc. 79 (1955), 173-215.

2. S. Bochner, Harmonic analysis and the theory of probability, Univ. of California Press, Berkeley, Calif., 1955.

3. K. L. Chung and W. H. J. Fuchs, On the distribution of values of sums of random variables, Mem. Amer. Math. Soc. No. 6 (1951), 12 pp.

4. R. M. Dudley, Random walks on abelian groups, Proc. Amer. Math. Soc. 13 (1962), 447-450.

5. P. R. Halmos, Measure theory, Van Nostrand, Princeton, N. J., 1950.

6. E. Marczewski and P. Sikorski, Measures in nonseparable metric spaces, Colloq. Math. 1 (1948), 133-139.

7. L. S. Pontrjagin, Topological groups, Princeton Univ. Press, Princeton, N. J., 1946.

8. Karl Stromberg, $A$ note on the convolution of regular measures, Math. Scand. 7 (1959), 347-352.

University of California, Berkeley 